\title{
ADAPTIVE PARTIAL STABILIZATION, LIMIT DYNAMICS AND BIFURCATION ANALYSIS
}

\author{
Gholam Reza Rokni Lamooki
}

\begin{abstract}
A class of autonomous control systems with fixed unknown parameters is considered to be stabilized with respect to only a part of the variables. A certain type of such systems can be recursively adaptively partially stabilized. The bifurcation analysis reveals the nature of the closed loop system.
\end{abstract}

\section{Introduction}

We consider the class of adaptive control systems with some fixed but unknown parameters. There are two general approaches to deal with such uncertainties which are known as indirect and direct methods; see [2], [6], and [9] for more details. We adopt the direct method where the actual values of the unknown parameters are not the objective of the control; see [10] as an example of the technique in the class of universal adaptive control. In presence of persistence of excitation these estimations converge to the actual value of the unknown parameters. However, in absence of persistence of excitation, they do not necessarily converge to the actual values of those unknown parameters. In general, such estimations may not converge to any value. As stated earlier, when the unknown parameters are not the objective of the control, their estimations, which are adapted by tuning functions in absence of persistence of excitation, do not converge to the unknown parameters. They only stay bounded or at best converge to some values depending on initial conditions. This can be performed through Lyapunov based design. In Lyapunov direct method, the stability is proved by a positive definite Lyapunov function with negative definite time derivative along the solutions of the closed-loop system. When the time derivative of Lyapunov function is negative but not negative definite, LaSalle's invariance principle yields convergence to an invariant set. Deeper approaches, known as partial stability, concern the sign of Lyapunov function and its time derivative with respect to a part of the variables, where stability is investigated with respect to the given part of the variables; see [17],

Received March 15, 2011; Revised July 19, 2012.

2010 Mathematics Subject Classification. 93D15, 93D20, 93D21.

Key words and phrases. partial stability, adaptive control, backstepping, bifurcation. 
[19], and also [20] for a review. In partial stability, those variables which we do not intend to stabilize could be superfluous variables or phase variables; see [1], [16] and [19] for more details. We focus on a system with fixed but unknown parameters where the stability with respect to only a part of the variables is required.

The considered class of systems is, in strict feedback form, aimed to be stabilized with respect to only a part of the phase variables using tuning functions. Therefore the variables of the adaptive partially stabilized closed-loop system split into a stabilized part which converges to the partial equilibrium, and a non-stabilized part which stay bounded. This latter part of the variables consists of two groups. The first group corresponds to those variables which were not aimed, or were not possible, to be stabilized and may not converge to any value. The second group consists of parameters' estimation. When nonstabilized variables of the closed-loop system converge to some values; their limit values depend on the initial conditions. This leads to the existence of a manifold of equilibria, the limit controller, and the limit system. Each equilibrium point on this manifold has its own stability properties and corresponds to a limit system. A single-wedge bifurcation occurs when an equilibrium possesses a non-empty interior basin of attraction while its corresponding limit system is unstable. We investigate the bifurcation diagrams for the case when the original system is three-dimensional with one stabilizable variable, two nonstabilizable variables and one unknown parameter. It will be proved that in this four dimensional closed-loop system single-wedge bifurcation will not take place.

Consider the nonlinear system

$$
\dot{x}=f(x), \quad f(0)=0, \quad x=\left(y_{1}, \ldots, y_{p}, z_{1}, \ldots, z_{q}\right)=(y, z),
$$

where $p>0, q \geq 0$ and $n=p+q$. Suppose that system (1) is smooth and its solutions uniquely exist for all time for initial conditions in a domain

$$
\|y\| \leq H,\|z\|<\infty
$$

for some $H>0$.

Definition 1.1 ([19]). The function $V: D \rightarrow \mathbb{R}$ is called $y$-positive definite if there exists a continuous function $W(y)$ which is positive for $y \neq 0$ in a domain (2) with $W(0)=0$, such that $V(y, z) \geq W(y)$.

Definition $1.2([19])$. The origin of system (1) is called $y$-stable if for any $\epsilon>0$ there exists $\delta>0$ such that for all initial conditions $x_{0}$ with $\left\|x_{0}\right\|<\delta$ the solution $y(t)$ satisfies $\|y(t)\|<\epsilon$. The system (1) is called asymptotically $y$-stable if, in addition, there exists a number $\Delta>0$ such that for all initial conditions $x_{0}$ with $\left\|x_{0}\right\|<\Delta$ the solution $y(t)$ satisfies $\lim _{t \rightarrow \infty} y(t)=0$. The system (1) is called globally asymptotically $y$-stable if the domain (2) is replaced with $\|x\|<\infty$ and the convergence $\lim _{t \rightarrow \infty} y(t)=0$ holds for arbitrary $x_{0}$. 
The notion of $y$-stable, sometimes, is addressed as stable with respect to $y$. According to [16], for the system (1), there is a $y$-positive definite $V$-function such that $\dot{V} \leq 0$ in a domain (2), then the origin of the system (1) is $y$-stable. We recall the following boundedness condition.

Definition 1.3 ([1], [19]). The vector field of system (1) is called $z$-bounded if the component $z(t)$ of its solutions stay bounded.

Theorem 1.4 ([13]). Suppose that the system (1) is z-bounded. Assume that there is a $V$-function for the system (1) defined on $\|x\| \leq H$ for some $H>0$. Let $M_{1}=\{x \mid \dot{V}(x)=0\}, M_{2}=\{x \mid y=0\}$ and assume that $\dot{V}(x)<0$ for $x \notin M_{1}$. Suppose $M_{2}$ is invariant and $M=M_{1} \backslash M_{2}$ contains no entire positive semi trajectory in the domain $\|x\|<H$. Then, the system (1) is asymptotically $y$-stable. If these conditions are satisfied in the domain $\|x\|<\infty$ and the corresponding $V$-function is radially $y$-unbounded; that is $\lim _{\|y\| \rightarrow \infty} W(y)=$ $\infty$, then the system (1) is globally asymptotically $y$-stable.

It is proved in [12] that these results may not be true if $z$-boundedness fails; see [11] for other methods of stabilization. This paper is organized as follows. In Section 2 the main problem will be established. In Section 3 the stabilization process will be stated. The bifurcation analysis will be carry out in Sections 45. The possibility of occurrence of bad behavior for the closed-loop system will be discussed in Section 6 . The paper will be concluded by a brief discussion.

\section{Problem statement}

Consider the system

$$
\dot{x}=f\left(x, u, \theta^{*}\right) .
$$

Here $x=(y, z) \in \mathbb{R}^{n}$ is the state variables, where $y \in \mathbb{R}^{p}, z \in \mathbb{R}^{q}, p>0, q \geq 0$, $p+q=n, u \in \mathbb{R}^{s}$ is the input and $\theta^{*} \in \mathbb{R}^{\ell}$ is a vector of fixed but unknown parameters. We assume independence of $\theta^{*}$; that is, $x=0$ is an equilibrium of the unforced system, so that $f\left(0,0, \theta^{*}\right)=0$ for all $\theta^{*}$. An adaptive controller which stabilizes the system (3) with respect to $y$ about the equilibrium $x=0$ takes the form $u=g(x, \hat{\theta})$, where $g(0, \hat{\theta})=0$ for all $\hat{\theta}$, and the parameter $\hat{\theta}$ is adapted according to $\dot{\hat{\theta}}=G(x, \hat{\theta})$. Adaptive partial stabilization requires that each solution of the adaptive closed-loop system satisfies $y(t) \rightarrow 0$ as $t \rightarrow \infty$ while $z$ and $\hat{\theta}$ stay bounded. To illustrate adaptive backstepping control, consider a three-dimensional system, $x=(y, z) \in \mathbb{R}^{2}, w \in \mathbb{R}, u \in \mathbb{R}$ with a fixed unknown parameter $\theta^{*}$.

$$
\left\{\begin{array}{l}
\dot{x}=f_{1}\left(x, w, \theta^{*}\right), \\
\dot{w}=f_{2}(x, w, u) .
\end{array}\right.
$$

Adaptive backstepping means that one first finds a feedback $w=\kappa(x, \hat{\theta})$ and an intermediate parameter adaptation $\hat{\theta}=G_{1}(x, \hat{\theta})$ so that, in the first step, 
the closed-loop system

$$
\left\{\begin{array}{l}
\dot{x}=f_{1}\left(x, \kappa(x, \hat{\theta}), \theta^{*}\right), \\
\dot{\hat{\theta}}=G_{1}(x, \hat{\theta})
\end{array}\right.
$$

is $y$-stabilized, that is, $y(t) \rightarrow 0$ as $t \rightarrow \infty$ while $z$ and $\hat{\theta}$ stay bounded. In the next step, we utilize the change of variable $\zeta=w-\kappa$ and find the dynamics of $\zeta$ as $\dot{\zeta}=v=u+H(x, \zeta, \hat{\theta})$. Then, we specify the controller $u=g(x, \zeta, \hat{\theta})$ and the parameter adaptation law $\dot{\hat{\theta}}=G(x, \zeta, \hat{\theta})$ so that $\zeta(t)=w(t)-\kappa(x, \hat{\theta}) \rightarrow 0$ and $y(t) \rightarrow 0$ as $t \rightarrow \infty$ while $z$ and $\hat{\theta}$ stay bounded.

Now we focus on the question of determining parameters which necessarily converge. In the process of adaptive stabilization, the parameter estimation $\hat{\theta}$ does not need to converge to the actual unknown parameter $\theta^{*}$. In general, $\hat{\theta}(t)$ does not need to converge at all. It is only required that for any initial conditions of the closed-loop system, the parameter estimation $\hat{\theta}(t)$ stay bounded with a bound depends on initial conditions. However, in many practical cases, the convergence of the parameter estimation is either desirable or a requested aim for the control design. When this is the case, for any given initial conditions of the closed-loop system, the parameter estimation $\hat{\theta}(t)$ converges to a limit value let say $\theta_{\infty}$. This limit value defines a limit controller $u_{\infty}=g\left(x, \zeta, \hat{\theta}_{\infty}\right)$ corresponding to a limit system. When the system gets enough near this limit system, one would turn off the adaptation and let the system goes under the dynamics of this non-adaptive closed-loop system; see [7] for more details concerning this idea of frozen-time controller. This is fine as far as the limit system is stabilized. Surprisingly, the limit system may not be stabilized; see [18]. The worth situation arises when there exists a destabilized limit system with a non empty interior basin of attraction. We discuss this issue in Section 6 .

In some problems, the actual value of the unknown parameters are required to be detected. This means that the parameters' estimation should converge to a value equal to the actual values of the unknown parameters. The adaptive control does not guarantee such convergence; in fact, this should be carried out by considering persistence of excitation along adaptive stabilization; see [6] for more details.

Adaptive backstepping can be used for systems in strict feedback form with some fixed unknown parameters. A nonlinear system in strict feedback form with a vector of fixed but unknown parameters is thoroughly studied in [8]; see also [18]. The concept of the strict feedback form can be extended to the class of partially stabilizable systems where the stability with respect to only a part of the variables is the objective of the control in presence of some unknown parameters. For each $1 \leq i \leq r$ let $\bar{x}_{i}=\left[x_{1}, \ldots, x_{i}\right]^{T}$ and $x_{i}=\left[y_{i}^{T} z_{i}^{T}\right]^{T} \in$ $\mathbb{R}^{p_{i}+q_{i}}$. We define the general form of strict feedback form by

$$
\dot{x}_{i}=B^{i} x_{i+1}+\varphi_{i}\left(\bar{x}_{i}\right) \theta^{*},
$$


where $p_{i}+q_{i}=m_{i}$ for $1 \leq i \leq r$. We denote $\bar{m}_{i}=\sum_{j=1}^{i} m_{j}$ for each $1 \leq i \leq r$. Let $w \in \mathbb{R}^{s}, \theta^{*} \in \mathbb{R}^{\ell}$ be a vector of unknown parameters, $\dot{w}=u+\varphi(x, w) \theta^{*}$, where $\varphi_{i}: \mathbb{R}^{\bar{m}_{i}} \rightarrow \mathbb{R}^{m_{i}} \times \mathbb{R}^{\ell}$ with $\varphi_{i}(0)=0$ for all $1 \leq i \leq r, \varphi: \mathbb{R}^{\bar{m}_{r}+s} \rightarrow$ $\mathbb{R}^{s} \times \mathbb{R}^{\ell}$, and $u \in \mathbb{R}^{s}$ is the controller. For each $1 \leq i \leq r$ we have

$$
\left\{\begin{array}{l}
\dot{y}_{i}=B_{11}^{i} y_{i+1}+B_{12}^{i} z_{i+1}+\varphi_{1}^{i}\left(\bar{y}_{i}, \bar{z}_{i}\right) \theta^{*} \\
\dot{z}_{i}=B_{21}^{i} y_{i+1}+B_{22}^{i} z_{i+1}+\varphi_{2}^{i}\left(\bar{y}_{i}, \bar{z}_{i}\right) \theta^{*}
\end{array}\right.
$$

Here $\bar{y}_{i}=\left[\begin{array}{llll}y_{1} & y_{2} & \cdots & y_{i}\end{array}\right]^{T}, \bar{z}_{i}=\left[z_{1} z_{2} \cdots z_{i}\right]^{T}, y=\left[y_{1} y_{2} \cdots y_{r}\right]^{T}$, and $z=$ $\left[z_{1} z_{2} \cdots z_{r}\right]^{T}$. In Section 3, we consider the system (4) in form of (6) and (7).

\section{Stabilization process}

Consider the following system

$$
\left\{\begin{array}{c}
\dot{x}=B w+\theta^{*} \varphi(x): \\
\dot{y}=b w+\varphi_{1}(y, z) \theta^{*}, \\
\dot{z}=c w+\varphi_{2}(y, z) \theta^{*}, \\
\dot{w}=u
\end{array}\right.
$$

To fit into the form (7), here $x=[y, z]^{T} \in \mathbb{R}^{2}, B=[b, c]^{T}, \varphi=\left[\varphi_{1}, \varphi_{2}\right]^{T}$ is smooth with $\varphi(0)=0$, and $\theta^{*}$ is an unknown fixed scalar parameter; see [14]. Under some conditions on $\varphi$, the adaptive partial stabilization of system (8) with respect to $y$ can be achieved through a recursive design. In summary, the stabilization is performed in two steps: first, we stabilize the $x$-equation with respect to $y$ by assuming that $w$ is a controller, which leads to the requirement $w=\kappa(x, \hat{\theta})=-b^{-1}\left(\hat{\theta} \varphi_{1}+h(y)\right)$ where $\hat{\theta}$ is the estimation for $\theta^{*}$ and $h$ is a scalar real valued function satisfying $h(0)=0$ and $y h(y)>0$ for all $y \neq 0$. Next, we stabilize both $y$ and $\zeta=w-\kappa(x, \hat{\theta})=w+b^{-1}\left(\hat{\theta} \varphi_{1}+h(y)\right)$ using an appropriate controller $u=u(y, w)$ and parameter estimation. The construction is explained in details as follows and the stability properties of the closed-loop system will be discussed in Theorems 3.2-3.3.

In order to proceed with full details of stabilization, we let $\theta^{*}=\hat{\theta}+k$, where $\hat{\theta}$ and $k$ are the estimation for the unknown parameter and the error of estimation respectively. We choose $V_{1}=\frac{1}{2}\left(y^{2}+k^{2}\right)$ as the first step $V$-function. We have

$$
\dot{V}_{1}=y \dot{y}+k \dot{k}=y\left[b w+\hat{\theta} \varphi_{1}\right]+k\left[-\dot{\hat{\theta}}+y \varphi_{1}\right]
$$

Assuming $b \neq 0$, we can choose

$$
w=\kappa(x, \hat{\theta})=-b^{-1}\left(\hat{\theta} \varphi_{1}+h(y)\right), \dot{\hat{\theta}}=y \varphi_{1} .
$$


This leads to $\dot{V}_{1}=-y h(y)$, and the following intermediate close-loop system is achieved.

$$
\left\{\begin{array}{l}
\dot{y}=-h(y)+k \varphi_{1}, \\
\dot{z}=-c b^{-1} h(y)+c b^{-1} k \varphi_{1}+\theta^{*}\left(\varphi_{2}-c b^{-1} \varphi_{1}\right), \\
\dot{k}=-y \varphi_{1} .
\end{array}\right.
$$

The intermediate close-loop system (10) necessarily needs to be stabilized with respect to $y$. We discuss that later in this section. Now, we introduce a new variable $\zeta=w-\kappa(x, \hat{\theta})=w+b^{-1}\left(\hat{\theta} \varphi_{1}+h(y)\right)$. Then we have $\dot{\zeta}=v$, where

$$
\begin{aligned}
v:= & \dot{w}+b^{-1}\left(\dot{\hat{\theta}} \varphi_{1}+\left(\frac{\partial \varphi_{1}}{\partial y} \dot{y}+\frac{\partial \varphi_{1}}{\partial z} \dot{z}\right) \hat{\theta}+\dot{y} h^{\prime}(y)\right) \\
= & u+b^{-1} \varphi_{1} \dot{\hat{\theta}}+b^{-1} \hat{\theta}\left(\frac{\partial \varphi_{1}}{\partial y}+h^{\prime}(y)\right)\left(b \zeta+b k+\hat{\theta} \varphi_{1}+k \varphi_{1}\right) \\
& +b^{-1} \hat{\theta} \frac{\partial \varphi_{1}}{\partial z}\left(c \zeta+c k+\hat{\theta} \varphi_{2}+k \varphi_{2}\right) \\
= & u+b^{-1} \varphi_{1} \dot{\hat{\theta}}+b^{-1} \hat{\theta}\left(\frac{\partial \varphi_{1}}{\partial y}+h^{\prime}(y)\right)\left(b \zeta-\hat{\theta} \varphi_{1}-h(y)+\hat{\theta} \varphi_{1}+k \varphi_{1}\right) \\
& +b^{-1} \hat{\theta} \frac{\partial \varphi_{1}}{\partial z}\left(c \zeta-c b^{-1} \hat{\theta} \varphi_{1}-c b^{-1} h(y)+\hat{\theta} \varphi_{2}+k \varphi_{2}\right) .
\end{aligned}
$$

By utilizing this new variable, we can reorganize the original equations (8) as follows

$$
\left\{\begin{array}{l}
\dot{y}=b(\zeta+k)+\hat{\theta} \varphi_{1}+k \varphi_{1}, \\
\dot{z}=c(\zeta+k)+\hat{\theta} \varphi_{2}+k \varphi_{2}, \\
\dot{\zeta}=v
\end{array}\right.
$$

We choose the $V$-function as $V=V_{1}+\frac{1}{2} \zeta^{2}$, then we have

$$
\begin{aligned}
\dot{V}= & y \dot{y}+k \dot{k}+\zeta \dot{\zeta} \\
= & y\left(b \zeta-h(y)+k \varphi_{1}\right)+k \dot{k} \\
& +\zeta\left(u+b^{-1} \varphi_{1} \dot{\hat{\theta}}+b^{-1} \hat{\theta}\left(\frac{\partial \varphi_{1}}{\partial y}+h^{\prime}(y)\right)\left(b \zeta-\hat{\theta} \varphi_{1}-h(y)+\hat{\theta} \varphi_{1}\right)\right. \\
& \left.+b^{-1} \hat{\theta} \frac{\partial \varphi_{1}}{\partial z}\left(c \zeta-c b^{-1} \hat{\theta} \varphi_{1}-c b^{-1} h(y)+\hat{\theta} \varphi_{2}\right)\right) \\
& +\zeta\left(b^{-1} \hat{\theta}\left(\frac{\partial \varphi_{1}}{\partial y}+h^{\prime}(y)\right) \varphi_{1}+b^{-1} \hat{\theta} \frac{\partial \varphi_{1}}{\partial z} \varphi_{2}\right) k
\end{aligned}
$$

We can rewrite $\dot{V}$ as follows

$$
\dot{V}=-y h(y)
$$




$$
\begin{aligned}
& +\zeta\left(u+b y+b^{-1} \varphi_{1} \dot{\hat{\theta}}+b^{-1} \hat{\theta}\left(\frac{\partial \varphi_{1}}{\partial y}+h^{\prime}(y)\right)(b \zeta-h(y))\right. \\
& \left.+b^{-1} \hat{\theta} \frac{\partial \varphi_{1}}{\partial z}\left(c \zeta-c b^{-1} \hat{\theta} \varphi_{1}-c b^{-1} h(y)+\hat{\theta} \varphi_{2}\right)\right) \\
& +k\left[\zeta\left(b^{-1} \hat{\theta}\left(\frac{\partial \varphi_{1}}{\partial y}+h^{\prime}(y)\right) \varphi_{1}+b^{-1} \hat{\theta} \frac{\partial \varphi_{1}}{\partial z} \varphi_{2}\right)-\dot{\hat{\theta}}+y \varphi_{1}\right]
\end{aligned}
$$

then by choosing

$$
\begin{aligned}
u= & -\left[b y+b^{-1} \varphi_{1} \dot{\hat{\theta}}+b^{-1} \hat{\theta}\left(\frac{\partial \varphi_{1}}{\partial y}+h^{\prime}\right)(b \zeta-h(y))\right. \\
& \left.+b^{-1} \hat{\theta} \frac{\partial \varphi_{1}}{\partial z}\left(c \zeta-c b^{-1} \hat{\theta} \varphi_{1}-c b^{-1} h(y)+\hat{\theta} \varphi_{2}\right)\right]-\mu(\zeta), \\
\dot{\hat{\theta}}= & \zeta\left(\frac{\hat{\theta}}{b}\left(\frac{\partial \varphi_{1}}{\partial y}+h^{\prime}\right) \varphi_{1}+\frac{\hat{\theta}}{b} \frac{\partial \varphi_{1}}{\partial z} \varphi_{2}\right)+y \varphi_{1},
\end{aligned}
$$

where $\mu$ is a scalar real valued function satisfying $\mu(0)=0$ and $\zeta \mu(\zeta)>0$ for all $\zeta \neq 0$. Then, we have $\dot{V}=-y h(y)-\zeta \mu(\zeta)$, and the auxiliary closed-loop system will become

$$
\left\{\begin{array}{l}
\dot{y}=-h(y)+b \zeta+k \varphi_{1} \\
\dot{\zeta}=-\mu(\zeta)+b^{-1}\left(\theta^{*}-k\right)\left(\frac{\partial \varphi_{1}}{\partial y}+h^{\prime}(y)\right) k \varphi_{1}+b^{-1}\left(\theta^{*}-k\right) \frac{\partial \varphi_{1}}{\partial z} k \varphi_{2} \\
\dot{z}=c \zeta-c b^{-1}\left(\theta^{*}-k\right) \varphi_{1}-c b^{-1} h(y)+\theta^{*} \varphi_{2} \\
\dot{k}=-\zeta\left(\frac{\hat{\theta}}{b}\left(\frac{\partial \varphi_{1}}{\partial y}+h^{\prime}(y)\right) \varphi_{1}+\frac{\left(\theta^{*}-k\right)}{b} \frac{\partial \varphi_{1}}{\partial z} \varphi_{2}\right)-y \varphi_{1}
\end{array}\right.
$$

The arguments of $\varphi_{1}, \varphi_{2}$ have been suppressed for clarity. In summary we have

(15) $\quad V_{1}=\frac{1}{2}\left(y^{2}+k^{2}\right) \geq a_{1}(\|(y, k)\|), \quad \dot{V}_{1}=-y h(y) \leq-c_{1}(|y|)$,

(16) $V=V_{1}+\frac{1}{2} \zeta^{2} \geq a(\|(y, k, \zeta)\|), \quad \dot{V}=-y h(y)-\zeta \mu(\zeta) \leq-c(\|(y, \zeta)\|)$,

for some monotonically increasing functions $a, c, a_{1}, c_{1}$.

Remark 3.1. The auxiliary closed-loop system (14) is asymptotically $y$-stable if $h(y)$ and $\varphi_{1}$ are bounded along its solutions [17]. This implies local asymptotic stability for the closed-loop system (14). In other words, for each $\epsilon>0$, there exists a number $\Delta>0$ such that for all initial conditions $\left(y_{0}, \zeta_{0}, z_{0}, k_{0}\right)$ with $\left\|\left(y_{0}, \zeta_{0}, z_{0}, k_{0}\right)\right\|<\Delta$ the norm of the solution is bounded by $\epsilon$ and $y(t)$ satisfies $\lim _{t \rightarrow \infty} y(t)=0$. This would be fine as long as one has some idea about the size of the actual value of $\theta^{*}$. However, generally, $\theta^{*}$ has no bound. Thus, we cannot consider any bound on $k$. That is we expect the existence of a number $\bar{\Delta}>0$ such that for all initial conditions $\left(y_{0}, \zeta_{0}, z_{0}, k_{0}\right)$ with $\left\|\left(y_{0}, \zeta_{0}, z_{0}\right)\right\|<$ $\bar{\Delta}$ and $k_{0} \in \mathbb{R}$ the norm of the solution is bounded by $\epsilon$ and $y(t)$ satisfies $\lim _{t \rightarrow \infty} y(t)=0$.

Now we are ready to state the main theorems. 
Theorem 3.2. Suppose in (8) we have $b \neq 0$, the $z$-component of system (14) is bounded, and $\varphi_{i}=y \psi^{i}$ for some smooth functions $\psi^{i}, i=1,2$, then the feedback controller (12) and the parameter adaptation (13) globally asymptotically stabilize the system (8) with respect to $(y, w)$. Moreover, the closed-loop system will have a globally attracting manifold of equilibria where each equilibrium corresponds to a limit system.

Proof. We need to show the asymptotic $y$-stability for the system (10) and asymptotic $(y, \zeta)$-stability for the system (14). First we focus on intermediate system (10) recalling equation (15). We let $M_{1}=\left\{(y, z, k) \mid \dot{V}_{1}=0\right\}, M_{2}=$ $\{(y, z, k) \mid y=0\}$, and $M=M_{1} \backslash M_{2}$. One can observe that $M_{2}$ is invariant and $M=\emptyset$ so that it cannot contain the entire positive semi trajectories. Thus, according to Theorem 1.4, the system (10) is asymptotically $y$-stable. This asymptotic stability is global since $V_{1}$ is radially $y$-unbounded.

Now, we focus on the auxiliary closed-loop system (14). Recalling equation (16), for the auxiliary system (14), we let

$$
M_{1}=\{x \mid \dot{V}=0\}=\{(y, \zeta, z, k) \mid y h(y)+\zeta \mu(\zeta)=0\} .
$$

The equation $y h(y)+\zeta \mu(\zeta)=0$ cannot have nonzero solution, because for $y \neq 0$ we have $y h(y)>0$ and for $\zeta \neq 0$ we have $\zeta \mu(\zeta)>0$. Again, we denote

$$
M_{2}=\{(0,0, z, k) \mid z, k \in \mathbb{R}\}, \quad M=M_{1} \backslash M_{2} .
$$

We observe that $M_{2}$ is invariant and $M=\emptyset$, so that $M$ cannot contain the entire positive semi trajectories. According to Theorem 1.4, the closedloop system $(14)$ is asymptotically $(y, \zeta)$-stable. In fact, $V$ is radially $(y, \zeta)$ unbounded, thus this asymptotic stability is global.

There is one more step to complete the proof. We need to utilize the standard backstepping technique by observing that the global convergence $\zeta \rightarrow 0$ guarantees that $\lim _{t \rightarrow \infty}|w(t)-\kappa(y(t), z(t), \hat{\theta}(t))|=0$. This reduces the system (14) to the system (10) which leads to the global convergence $y \rightarrow 0$. Therefore, we conclude that the closed-loop system (8) and (12)-(13) is globally asymptotically $y$-stable. We have $\kappa(0, z, \hat{\theta})=0$, then the closed-loop system (8) and (12)-(13) is asymptotically stabilized with respect to $(y, w)$. The global attracting manifold of equilibria for the auxiliary closed-loop system $(14)$ is $(z, k)$-plane, where $(y, \zeta)=(0,0)$. Consequently, the closed-loop system (8) and (12)-(13) has a manifold of equilibria which is $(z, k)$-plane, where $(y, w)=(0,0)$. This implies that two variables $z(t)$ and $k(t)$ converges to some values depending on initial conditions. The limit value of $k$ defines a limit controller which accordingly defines a limit system.

If we do not assume that $\varphi_{i}=y \psi^{i}, i=1,2$, then $M_{2}$ in Theorem 3.2 will not be necessarily invariant. Therefore, $(y, w)$-stability is not the case and the global attracting manifold of equilibria does not exist. However, the following theorem which directly uses $V$-functions, proves $y$-stability for the closed-loop system. 
Theorem 3.3. Suppose in (8) we have $b \neq 0$ and the $z$-component of system (14) is bounded, then the feedback controller (12) and the parameter adaptation (13) globally asymptotically stabilize the system (8) with respect to $y$.

Proof. We need to show the asymptotic $y$-stability for the system (10) and asymptotic $(y, \zeta)$-stability for the system (14). First we focus on intermediate system (10). According to equation (15),

$$
\|\left(y\left(t ; y_{0}, z_{0}, k_{0}\right), k\left(t ; y_{0}, z_{0}, k_{0}\right)\|\leq\|\left(y_{0}, k_{0}\right) \|, \quad t \geq 0 .\right.
$$

This shows that the cylinder $\|(y, k)\| \leq H,|z|<\infty$ for any $H>0$, is invariant. Note that any bound $\bar{H}>0$ on $z(t)$ depends on $\left(y_{0}, z_{0}, k_{0}\right)$; therefore, we do not have any bounded invariant cylinder. $V_{1}$ is a non increasing function; as a result, $\lim _{t \rightarrow \infty} V_{1}(t)=V_{1}^{*}$ exists for some $V_{1}^{*} \geq 0$. Suppose $(y(t), k(t))$ be the projection of $(y(t), z(t), k(t))$ on a compact disk $\|(y, k)\| \leq H, z=z^{*}$ for some $z^{*} \in \mathbb{R}$. This projection is useful since $V_{1}$ does not depend on $z$. For any sequence $\left(t_{n}\right)$ with $\lim _{n \rightarrow \infty} t_{n}=\infty$ there is a subsequence $\left(t_{n_{j}}\right)$ and $\left(y^{*}, k^{*}\right)$ such that $\lim _{j \rightarrow \infty}\left(y\left(t_{n_{j}}\right), k\left(t_{n_{j}}\right)\right)=\left(y^{*}, k^{*}\right)$. Since $V_{1}$ is continuous, we have $V_{1}^{*}=\lim _{j \rightarrow \infty} V_{1}\left(t_{n_{j}}\right)=\lim _{j \rightarrow \infty} V_{1}\left(y\left(t_{n_{j}}\right), k\left(t_{n_{j}}\right)\right)=V_{1}\left(y^{*}, k^{*}\right)$. This implies that

$$
\dot{V}_{1}\left(y^{*}, k^{*}\right)=0 .
$$

Therefore, we have $y^{*}=0$. Since $\left(y\left(t_{n_{j}}\right), k\left(t_{n_{j}}\right)\right)$ is the projection of $\left(y\left(t_{n_{j}}\right)\right.$, $\left.k\left(t_{n_{j}}\right), z\left(t_{n_{j}}\right)\right)$ on an arbitrary disk $\|(y, k)\| \leq H, z=z^{*}$ and converges to the point $\left(y^{*}, k^{*}\right)=\left(0, k^{*}\right)$, then

$$
\omega_{\left(y_{0}, z_{0}, k_{0}\right)} \subset\{(y, z, k)|y=0,| k \mid \leq H, z \in \mathbb{R}\},
$$

where, $\omega_{\left(y_{0}, z_{0}, k_{0}\right)}$ denotes the $\omega$-limit set of $\left(y_{0}, z_{0}, k_{0}\right)$. Hence, $y(t)$ converges to 0 while $k(t)$ and $z(t)$ are bounded.

Now, we focus on the closed-loop system (14). According to equation (16), for all $t \geq 0$, we have

(21) $\|\left(y\left(t ; y_{0}, \zeta_{0}, z_{0}, k_{0}\right), \zeta\left(t ; y_{0}, \zeta_{0}, z_{0}, k_{0}\right), k\left(t ; y_{0}, \zeta_{0}, z_{0}, k_{0}\right)\|\leq\|\left(y_{0}, \zeta_{0}, k_{0}\right) \|\right.$.

This shows that the cylinder $\|(y, \zeta, k)\| \leq H,|z|<\infty$ for any $H>0$, is invariant. Again, we need to note that any bound $\bar{H}>0$ on $z(t)$ depends on $\left(y_{0}, \zeta_{0}, z_{0}, k_{0}\right)$; therefore, we do not have any bounded invariant cylinder. $V$ is a non decreasing function; as a result, $\lim _{t \rightarrow \infty} V(t)=V^{*}$ exists for some $V^{*} \geq 0$. Suppose $(y(t), \zeta(t), k(t))$ be the projection of $(y(t), \zeta(t), z(t), k(t))$ on a compact ball $\|(y, \zeta, k)\| \leq H, z=z^{*}$ for some $z^{*} \in \mathbb{R}$. Again, this projection is useful since $V$ does not depend on $z$. For any sequence $\left(t_{n}\right)$ with $\lim _{n \rightarrow \infty} t_{n}=\infty$ there is a subsequence $\left(t_{n_{j}}\right)$ and $\left(y^{*}, \zeta^{*}, k^{*}\right)$ such that $\lim _{j \rightarrow \infty}\left(y\left(t_{n_{j}}\right), \zeta\left(t_{n_{j}}\right), k\left(t_{n_{j}}\right)\right)=\left(y^{*}, \zeta^{*}, k^{*}\right)$. We have $V^{*}=\lim _{j \rightarrow \infty} V\left(t_{n_{j}}\right)=$ $\lim _{j \rightarrow \infty} V\left(y\left(t_{n_{j}}\right), \zeta\left(t_{n_{j}}\right), k\left(t_{n_{j}}\right)\right)=V\left(y^{*}, \zeta^{*}, k^{*}\right)$ since $V$ is continuous. This implies that $\dot{V}\left(y^{*}, \zeta^{*}, k^{*}\right)=0$. Therefore, we have $y^{*}=\zeta^{*}=0$. Since $\left(y\left(t_{n_{j}}\right), \zeta\left(t_{n_{j}}\right), k\left(t_{n_{j}}\right)\right)$ is the projection of $\left(y\left(t_{n_{j}}\right), \zeta\left(t_{n_{j}}\right), z\left(t_{n_{j}}\right), k\left(t_{n_{j}}\right)\right)$ on an 
arbitrary ball $\|(y, \zeta, k)\| \leq H, z=z^{*}$ and converges to the point $\left(y^{*}, \zeta^{*}, k^{*}\right)=$ $\left(0,0, k^{*}\right)$, then

$$
\omega_{\left(y_{0}, \zeta_{0}, z_{0}, k_{0}\right)} \subset\{(y, \zeta, z, k)|y=\zeta=0,| k \mid \leq H, z \in \mathbb{R}\}
$$

where, $\omega_{\left(y_{0}, \zeta_{0}, z_{0}, k_{0}\right)}$ denotes the $\omega$-limit set of $\left(y_{0}, \zeta_{0}, k_{0}\right)$. Hence, $(y(t), \zeta(t))$ converges to 0 while $k(t)$ and $z(t)$ are bounded.

It is worth noting that $V_{1}$ is radially $y$-unbounded and $V$ is radially $(y, \zeta)$ unbounded. Therefore, the systems (10) is globally asymptotically $y$-stable and the system (14) is globally asymptotically $(y, \zeta)$-stable.

One more time, we need to utilize the standard backstepping technique by observing that the global convergence $\zeta \rightarrow 0$ guarantees that

$$
\lim _{t \rightarrow \infty}|w(t)-\kappa(y(t), z(t), \hat{\theta}(t))|=0 .
$$

This reduces the system (14) to the system (10) which leads to the global convergence $y \rightarrow 0$. Therefore, we conclude that the closed-loop system (8) and (12)-(13) is globally asymptotically $y$-stable.

We do not necessarily have $\kappa(0, z, \hat{\theta})=0$, then the closed-loop system (8) and (12)-(13) is not necessarily asymptotically stabilized with respect to $(y, w)$.

Remark 3.4. The convergence of parameters' adaptation, $\hat{\theta}(t)$, and the convergence of non stabilized variable, $z(t)$, in the closed-loop system (14) are significant issues. We have seen that the two-dimensional manifold of equilibria exists when $\varphi_{i}(y, z)=y \psi^{i}(y, z), i=1,2$. This manifold of equilibria has to be globally attracting. Nevertheless, every single equilibrium will have its own stability properties and its own local behavior. The bifurcation analysis of this manifold of equilibria will reveal stability properties of each equilibrium. Such properties are expected to be sensitive with respect to the actual value of the unknown parameter. The relevant bifurcation analysis for the closed-loop system (14) will be presented in Sections 4-5. The limit system mentioned in Theorem 3.2 is defined with the limit non adaptive controller characterized by the limit value of $k$. For practical purpose, when the adaptive closed-loop system gets near the equilibrium, one might consider the adaptation process to be switched off. If the limit system is generically stabilized, then the non adaptive closed-loop system will work until a perturbation from the equilibrium occurs. Unfortunately, such limit system is not guaranteed to be generically stabilized. In this case, cutting off the adaptation may leads to a serious failure of the designed control system. Full understanding of this issue requires the stability analysis and related possible bifurcations' studies for the limit systems. In Section 6 , the possibility of occurrence of such situation will be discussed. Existence of limit values for $k$ and $z$ is not guaranteed under the conditions of Theorem 3.3. 


\section{Eigenvalue analysis}

According to Theorem 3.2, when $\varphi_{i}(y, z)=y \psi^{i}(y, z), i=1,2$, the closedloop system (14) has a plane of equilibria, that is, $(y, \zeta)=0$ or equivalently $(z, k)$-plane. According to the aim of stabilization, this plane of equilibria is globally attracting. However, all equilibria are not necessarily stable and each equilibrium has its own stability properties. In this section and Section 5 , the plane of equilibria of the system (14), under the condition of Theorem 3.2, will be studied from bifurcation point of view. The relevant notion of bifurcation is bifurcation without parameters; see for example [4]. Suppose $h_{1}=h^{\prime}(0)$ and $\mu_{1}=\mu^{\prime}(0)$. Obviously, $h_{1}>0$ and $\mu_{1}>0$. Then, the linearization matrix of the auxiliary closed-loop system (14) around its arbitrary equilibrium $(k, z, y, \zeta)=\left(k_{\infty}, z_{\infty}, 0,0\right):=\chi_{\infty}$ is

$$
A_{\infty}=\left[\begin{array}{cccc}
0 & 0 & 0 & 0 \\
0 & 0 & g^{\infty} & c \\
0 & 0 & -h_{1}+k_{\infty} \psi_{\infty} & b \\
0 & 0 & g_{\infty} & -\mu_{1}
\end{array}\right],
$$

where,

$$
\begin{aligned}
g_{\infty} & =\left[k_{\infty} \psi_{\infty}\left(\psi_{\infty}+h_{1}\right)\right] \theta^{*}-\left[k_{\infty}^{2} \psi_{\infty}\left(\psi_{\infty}+h_{1}\right)\right], \\
g^{\infty} & =\left[\psi^{\infty}-c b^{-1} \psi_{\infty}\right] \theta^{*}-\left[c b^{-1}\left(h_{1}-k_{\infty} \psi_{\infty}\right)\right] .
\end{aligned}
$$

Here, $\psi_{\infty}=\psi^{1}\left(0, z_{\infty}\right)$ and $\psi^{\infty}=\psi^{2}\left(0, z_{\infty}\right)$. This matrix has two zero eigenvalues corresponding to the plane of equilibria. The other two eigenvalues which determine the stability properties of the linearization are given by roots of the characteristic polynomial

$$
P(\lambda)=\lambda^{2}+\left(h_{1}+\mu_{1}-k_{\infty} \psi_{\infty}\right) \lambda+\mu_{1}\left(h_{1}-k_{\infty} \psi_{\infty}\right)-g_{\infty} .
$$

The characteristic polynomial (26) has the quadratic form. Therefore, there are three critical cases: a single zero eigenvalue, a pair of purely imaginary eigenvalues, and a pair of zero eigenvalues; see [5] for more details on bifurcation theory.

Single zero eigenvalue

A simple algebra shows that the critical case of single zero eigenvalue is corresponding to $P(0)=0$ while $P^{\prime}(0) \neq 0$. These require

$$
g_{\infty} \neq-\mu_{1}^{2}, \quad k_{\infty} \psi_{\infty}+\mu_{1}^{-1} g_{\infty}=h_{1} .
$$

The remained non zero eigenvalue is $\lambda^{\prime}=-\left(\mu_{1}+\mu_{1}^{-1} g_{\infty}\right)$. When $\lambda^{\prime}>0$, the equilibrium $\chi_{\infty}$ is unstable; however, when $\lambda^{\prime}<0$ the stability properties of equilibrium $\chi_{\infty}$ depend on dynamics on center manifold. We denote the former by $T C_{2}$ and corresponds to $g_{\infty}<-\mu_{1}^{2}$ and the latter by $T C_{1}$ corresponds to $g_{\infty}>-\mu_{1}^{2}$. Suppose $g_{\infty}>-\mu_{1}^{2}$ and $k_{\infty} \psi_{\infty}+\mu_{1}^{-1} g_{\infty}=h_{1}+\epsilon$ for some small enough $\epsilon$. Then, the center manifold is trivial. The equilibrium $\chi_{\infty}$ is a saddle node for $\epsilon>0$ and is asymptotically stable for $\epsilon<0$. Now, suppose $g_{\infty}<-\mu_{1}^{2}$ 
and $k_{\infty} \psi_{\infty}+\mu_{1}^{-1} g_{\infty}=h_{1}+\epsilon$ for some small enough $\epsilon$. Then, the center manifold is again trivial. The equilibrium $\chi_{\infty}$ is a saddle node for $\epsilon>0$ and is a unstable node for $\epsilon<0$. This argument shows that both $T C_{1}$ and $T C_{2}$ are transcritical bifurcations from plane of equilibria.

A pair of purely imaginary eigenvalues

The critical case of a pair of purely imaginary eigenvalues $\lambda_{1,2}= \pm i \omega_{0}$ is corresponding to $P(0)=\omega_{0}^{2}>0$ while $P^{\prime}(0)=0$. These require

$$
\begin{aligned}
& h_{1}+\mu_{1}-k_{\infty} \psi_{\infty}=0, \\
& \mu_{1}\left(h_{1}-k_{\infty} \psi_{\infty}\right)-g_{\infty}>0 .
\end{aligned}
$$

Here $\omega_{0}=\sqrt{\mu_{1}\left(h_{1}-k_{\infty} \psi_{\infty}\right)-g_{\infty}}>0$. The intersection between the rootlocus of the eigenvalues $\lambda_{1,2}$ and the $\Im$-axis is generically transversal with respect to the variations of the parameters $h_{1}, \mu_{1}, k_{\infty}$. This can be easily checked using (26). These satisfy the necessary conditions for Hopf bifurcation, but for generic conditions and the direction of the Hopf bifurcation one needs to consider Hopf bifurcation from plane of equilibria and compute the first Lyapunov coefficients.

A pair of zero eigenvalues

The occurrence of a double zero eigenvalues requires that $k_{\infty}=k_{h}$ and $g_{\infty}=$ $-\mu_{1}^{2}$. This happens at the intersection of transcritical and Hopf bifurcation and is corresponding to Bogdanov-Takens bifurcation from plane of equilibria.

In next section, the bifurcation analysis will be presented along the assumptions and notions of Section 4.

\section{Bifurcation diagram}

In bifurcation with respect to parameters, the bifurcation diagram is usually built by foliation of the parameter space with copies of the phase space. The closed-loop system (14) under the assumption of Theorem 3.2 performs a two dimensional plane of equilibria. Any equilibrium on this plane of equilibria has its own stability properties depending on parameters. Therefore, the plane of equilibria can be partitioned into various regions each of which contains equilibria with topologically equivalent local neighborhoods. The boundary between regions are referred as bifurcation boundaries. Any diagram which clarify such boundaries can be considered as bifurcation diagram. The corresponding bifurcations are referred as bifurcation without parameters. Therefore, for any fixed value of the parameters we have a special pattern of bifurcation without parameters on the plane of equilibria. By varying the parameters of the system, the pattern of bifurcation without parameters on the plane of equilibria may change.

It is easy to see that any equilibrium $\chi_{\infty}$ satisfying $\psi_{\infty} k_{\infty}=0$ is asymptotically stable without any bifurcation. The equation $k_{\infty}=0$ indicates the $z_{\infty}$-axis in $\left(k_{\infty}, z_{\infty}\right)$-plane and equation $\psi_{\infty}=0$ means $\psi^{1}\left(0, z_{\infty}\right)=0$ indicating a family of lines parallel to $k_{\infty}$-axis. In summary, suppose $E=$ 


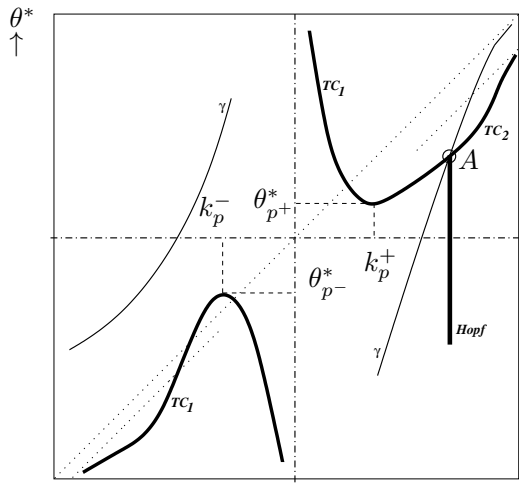

(a) $\rightarrow k_{\infty}$

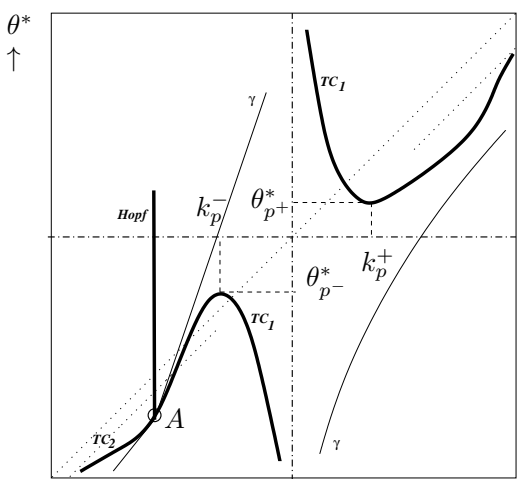

(b)

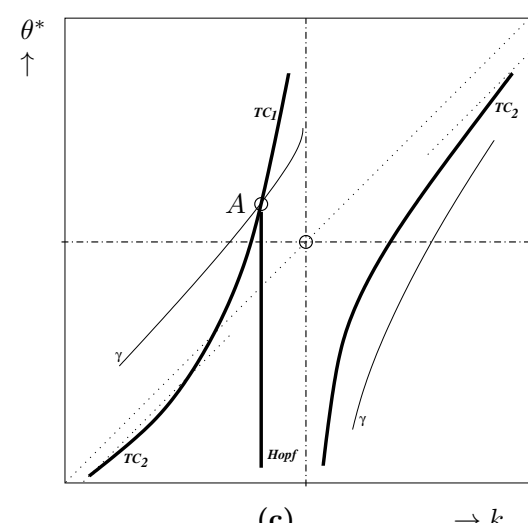

(c)

FiguRE 1. The bifurcation diagram using $\left(\psi_{\infty}, k_{\infty}, \theta^{*}\right)$ as bifurcation parameters. The occurrence of transcritical bifurcations and Hopf bifurcations in $\left(k_{\infty}, \theta^{*}\right)$-plane for three cases: (a) $\psi_{\infty}>0$, (b) $\psi_{\infty} \in\left(-\infty,-h_{1}\right)$, and (c) $\psi_{\infty} \in\left(-h_{1}, 0\right)$.

$\left\{z_{\infty} \mid \psi^{1}\left(0, z_{\infty}\right)=0\right\}$. Therefore, over the subset $\Xi=\left\{\chi_{\infty} \mid \psi_{\infty} k_{\infty}=0\right\}=$ $((\mathbb{R} \times E) \cup(\{0\} \times \mathbb{R})) \times\{0\} \times\{0\}$ of the plane of equilibria, all equilibria are asymptotically stable.

For $\psi_{\infty}=-h_{1}$, the occurrence of transcritical bifurcation requires $k_{\infty}=-1$ independent from the value of the unknown parameter $\theta^{*}$. In this case, we have $g_{\infty}=0$; thus, always $\lambda^{\prime}=-\mu_{1}<0$ which corresponds to transcritical bifurcation of type $T C_{1}$ and there will be no transcritical bifurcation of type $T C_{2}$. Also, there will be no equilibrium with purely imaginary eigenvalues since the equations (28)-(29) require $h_{1}+k_{\infty} h_{1}=-\mu_{1}<0$ and $h_{1}+k_{\infty} h_{1}>0$ which are impossible to be satisfied simultaneously. 
Now, suppose $\psi_{\infty}\left(\psi_{\infty}+h_{1}\right) \neq 0$. The transcritical bifurcation appears over the boundary defined by equation (27) and the Hopf bifurcation appears over the boundary defined by equations (28)-(29). By observing the equation (24), it is apparent that these boundaries are parametrized by the unknown parameter $\theta^{*}$. Variation of this unknown parameter has a significant effect on the pattern of the bifurcation without parameters in $\left(k_{\infty}, z_{\infty}\right)$-plane of equilibria. Therefore, we consider the three dimensional space $\left(k_{\infty}, z_{\infty}, \theta^{*}\right)$ as the extended space to construct the relevant bifurcation diagrams. A bifurcation diagram parametrized by $\theta^{*}$ can reveal many aspects of the underlying process. We perform a series of cross sections of the bifurcation diagram, in regions, where we expect to see similar situations. We consider three cross sections of the three dimensional bifurcation diagram in $\left(k_{\infty}, z_{\infty}, \theta^{*}\right)$-space at a fixed value of $z_{\infty}$ where $(1) \psi_{\infty}=\psi^{1}\left(0, z_{\infty}\right) \in(0,+\infty),(2) \psi_{\infty}=\psi^{1}\left(0, z_{\infty}\right) \in\left(-\infty,-h_{1}\right),(3)$ $\psi_{\infty}=\psi^{1}\left(0, z_{\infty}\right) \in\left(-h_{1}, 0\right)$.

Cross section at $z_{\infty}$ where $\psi_{\infty} \in(0,+\infty)$

First we focus on transcritical bifurcation. By substituting (24) into the inequality and equality of equation (27) and solving the resultant equation with respect to $\theta^{*}$ we get the following equations.

$$
\begin{aligned}
\theta^{*} & \neq \theta_{c}\left(k_{\infty}\right)=k_{\infty}-\frac{\mu_{1}^{2}}{\psi_{\infty}\left(\psi_{\infty}+h_{1}\right) k_{\infty}}, \\
\theta^{*} & =\left(k_{\infty}-\frac{\mu_{1}}{\psi_{\infty}+h_{1}}\right)+\frac{h_{1} \mu_{1}}{\psi_{\infty}\left(\psi_{\infty}+h_{1}\right) k_{\infty}} .
\end{aligned}
$$

For the fixed value of $z_{\infty}$ where $\psi_{\infty}>0$, the equation (31) is a curve in $\left(k_{\infty}, \theta^{*}\right)$-plane. This curve assumes a local minimum at the point $\left(k_{\infty}, \theta^{*}\right)=$ $\left(k_{p^{+}}, \theta_{p^{+}}^{*}\right)$ and a local maximum at $\left(k_{\infty}, \theta^{*}\right)=\left(k_{p^{-}}, \theta_{p^{-}}^{*}\right)$, where

$$
k_{p^{ \pm}}= \pm \sqrt{\frac{h_{1}}{\psi_{\infty}\left(\psi_{\infty}+h_{1}\right)}}, \quad \theta_{p^{ \pm}}^{*}=2 k_{p^{ \pm}}-\frac{\mu_{1}}{\psi_{\infty}+h_{1}} .
$$

Note that $\theta_{p^{+}}^{*}-\theta_{p^{-}}^{*}=2\left(k_{p^{+}}-k_{p^{-}}\right)=4 k_{p^{+}}>0$. Therefore, the local minimum is always above the local maximum. The following asymptotic property holds.

$$
\lim _{k_{\infty} \rightarrow \pm \infty}\left(\theta^{*}-k_{\infty}\right)=-\frac{\mu_{1}}{\psi_{\infty}+h_{1}} .
$$

It is easy to check that using inequality (30) we can define a strictly increasing curve $\gamma$ by $\theta^{*}=\theta_{c}\left(k_{\infty}\right)$ for a fixed $z_{\infty}$ where $\psi_{\infty}>0$. This curve intersect the curve defined by equation (31) at the point $A$ with coordinates $\left(k_{\infty}, \theta^{*}\right)=\left(k_{h}, \theta_{c}\left(k_{h}\right)\right)$, where $k_{h}=\left(\mu_{1}+h_{1}\right) / \psi_{\infty}>0$ and $\left.\theta_{c}\left(k_{h}\right)\right)>0$. For $k_{\infty}>0$, the transcritical bifurcation is of type $T C_{1}$ when $\theta^{*}>\theta_{c}\left(k_{h}\right)$ and is of type $T C_{2}$ when $\theta^{*}<\theta_{c}\left(k_{h}\right)$. For $k_{\infty}<0$, the transcritical bifurcation is of type $T C_{1}$ when $\theta^{*}<\theta_{c}\left(k_{h}\right)$ and is of type $T C_{2}$ when $\theta^{*}>\theta_{c}\left(k_{h}\right)$. 
Second, we focus on Hopf bifurcation. We substitute (24) into equality (28) and inequality (29). Then equations (28)-(29) reads as

$$
\begin{aligned}
& k_{\infty}=k_{h}=\frac{\mu_{1}+h_{1}}{\psi_{\infty}}, \\
& \theta^{*}<\theta_{c}\left(k_{h}\right) .
\end{aligned}
$$

Here, $k_{h}>0$ and $\theta_{c}\left(k_{h}\right)>0$. Where, for any fixed $z_{\infty}$ with $\psi_{\infty}>0$, the function $\theta_{c}$ is defined in equation (30). Using these arguments, we can sketch the bifurcation diagram for $\psi_{\infty}>0$ which is illustrated in Figure 1(a).

Cross section at $z_{\infty}$ where $\psi_{\infty} \in\left(-\infty,-h_{1}\right)$

The transcritical bifurcation is still described by equations (30)-(31). There is still the local maximum and minimum addressed by (32). The equation $\theta_{p^{+}}^{*}-\theta_{p^{-}}^{*}=2\left(k_{p^{+}}-k_{p^{-}}\right)=4 k_{p^{+}}>0$ holds; thus, the local minimum is always above the local maximum. Also, the asymptotic properties (33) holds. The curve $\gamma$ derived from inequality (30) which is defined by $\theta^{*}=\theta_{c}\left(k_{\infty}\right)$ is still a strictly increasing curve for a fixed $z_{\infty}$ where $\psi_{\infty}<-h_{1}$, but, now, this curve intersect the curve defined by equation (31) at the point $A$ with coordinates $\left(k_{\infty}, \theta^{*}\right)=\left(k_{h}, \theta_{c}\left(k_{h}\right)\right)$, where $k_{h}=\left(\mu_{1}+h_{1}\right) / \psi_{\infty}<0$ and $\left.\theta_{c}\left(k_{h}\right)\right)<0$. As the previous case, for $k_{\infty}>0$, the transcritical bifurcation is of type $T C_{1}$ when $\theta^{*}>\theta_{c}\left(k_{h}\right)$ and is of type $T C_{2}$ when $\theta^{*}<\theta_{c}\left(k_{h}\right)$. Also, for $k_{\infty}<0$, the transcritical bifurcation is of type $T C_{1}$ when $\theta^{*}<\theta_{c}\left(k_{h}\right)$ and is of type $T C_{2}$ when $\theta^{*}>\theta_{c}\left(k_{h}\right)$.

The Hopf bifurcation appears on half line defined by (34), where $\theta^{*}>\theta_{c}\left(k_{h}\right)$. Here, $k_{h}<0$ and $\theta_{c}\left(k_{h}\right)<0$. The bifurcation diagram of this case is illustrated in Figure 1(b).

Cross section at $z_{\infty}$ where $\psi_{\infty} \in\left(-h_{1}, 0\right)$

The transcritical bifurcation is still described by equations (30)-(31). However, now, this curve is strictly increasing. Also here, the curve $\gamma$ derived from inequality (30) which is defined by $\theta^{*}=\theta_{c}\left(k_{\infty}\right)$ is a strictly increasing curve for a fixed $z_{\infty}$ where $-h_{1}<\psi_{\infty}<0$. This curve intersect the curve defined by equation (31) at the point $A$ with coordinates $\left(k_{\infty}, \theta^{*}\right)=\left(k_{h}, \theta_{c}\left(k_{h}\right)\right)$, where $k_{h}=\left(\mu_{1}+h_{1}\right) / \psi_{\infty}<0$ and $\left.\theta_{c}\left(k_{h}\right)\right)<0$. In this case, for $k_{\infty}>0$, the transcritical bifurcation is of type $T C_{1}$ when $\theta^{*}<\theta_{c}\left(k_{h}\right)$ and is of type $T C_{2}$ when $\theta^{*}>\theta_{c}\left(k_{h}\right)$; and for $k_{\infty}<0$, the transcritical bifurcation is of type $T C_{1}$ when $\theta^{*}>\theta_{c}\left(k_{h}\right)$ and is of type $T C_{2}$ when $\theta^{*}<\theta_{c}\left(k_{h}\right)$.

The Hopf bifurcation appears on half line defined by (34), where $\theta^{*}>\theta_{c}\left(k_{h}\right)$. Here, $k_{h}<0$ and $\theta_{c}\left(k_{h}\right) \in \mathbb{R}$. The bifurcation diagram of this case is illustrated in Figure 1(c).

Bifurcation diagrams depicted in Figure 1 are typical and drawn using the general properties discussed in this section.

In next section, the limit system analysis will be presented along the assumptions and notions of Sections 4-5. 


\section{Limit system analysis}

In this section we consider adaptively partially stabilized system (14) under the assumptions of Theorem 3.2. The corresponding controller is designed to partially stabilize the system using the estimation of the unknown parameter. Such estimation is adapted automatically as part of the stabilization. One general drawback in adaptive control design is the possibility that the closed-loop limit system to be unstable. The worst situation is the existence of a destabilized limit system attracting a set of initial conditions with non empty interior. The identification, characterization and occurrence of such bad behavior in the adaptive stabilization of first and second order systems with one unknown parameter has been discussed in [15] where the phenomena of $n+p$-case is introduced for $n$ phase variables, all stabilized, and $p$-dimensional manifold of equilibria, coinciding with $p$ unknown parameters, of an $n+p$-dimensional closed-loop system. So far, there, the cases, $1+1$ and $2+1$ were discussed and the notion of single-wedge bifurcation were introduced. This notion is also addressed in [21] where the case $1+2$ is considered. The closed-loop system (14) under the assumption of Theorem 3.2 differs from the notion of $p+n$-cases. Because there are three phase variables and one unknown parameter in the original system (8), while the closed-loop system has a two dimensional manifold of equilibria. This is due to the fact that only a part of the phase variables is stabilized. The closed-loop system (14) can be roughly categorized as $2+2$-case, where there are a two dimensional manifold of equilibria of a four dimensional closed-loop system.

The single wedge bifurcation for an adaptive stabilized control system is defined as a bifurcation without parameter where the bifurcation point is an equilibrium corresponding to a destabilized limit system possessing a non empty interior basin of attraction. In this section we focus on the occurrence of singlewedge bifurcation. We consider the auxiliary closed-loop system for system (8), (12)-(13) which is given by (14), where without loss of generality we can assume $b=1$. The linearization of this system about an arbitrary equilibrium $(k, z, y, \zeta)=\left(k_{\infty}, z_{\infty}, 0,0\right)$ has two zero eigenvalues, $\lambda_{3}=\lambda_{4}=0$. There is a planar two-dimensional manifold of equilibria corresponding to these two zero eigenvalues. Over this manifold, we look for single-wedge bifurcation. That is an equilibrium with non empty interior basin of attraction with unstable limit system. It is assumed that in a neighborhood of that equilibrium other equilibria are either repelling or, otherwise, with stable limit system. One can observe that all transcritical bifurcations of type $T C_{2}$ have one positive eigenvalue $\lambda_{1}>0$, so that they will be repelling; as a result, they cannot perform any single-wedge bifurcation. However, all transcritical bifurcations of type $T C_{1}$ have corresponding eigenvalues $\lambda_{1}=0, \lambda_{2}<0$, so that their local behavior depend on corresponding three-dimensional center manifolds. A transcritical bifurcation of type $T C_{1}$ occurs at an equilibrium such as $(k, z, y, \zeta)=\left(k_{c}, z_{c}, 0,0\right)$ 
where $\mu_{1}\left(h_{1}-k_{c} \psi_{c}\right)-g_{c}=0$ with $\lambda_{1}=0$ and $\lambda_{2}=-h_{1}-\mu_{1}+k_{c} \psi_{c}<0$. Here, we have $\psi_{c}=\psi^{1}\left(0, z_{c}\right)$ and $g_{c}=k_{c}\left(\theta^{*}-k_{c}\right) \psi_{c}\left(\psi_{c}+h_{1}\right)$.

To perform the center manifold reduction of the system (14) around a typical transcritical bifurcation of type $T C_{1}$, the following change of variables is applied:

$$
K=k-k_{c}, \quad Z=z-z_{c} .
$$

The closed-loop system (14) is transformed to

$$
\left\{\begin{aligned}
\dot{K}= & -\zeta\left(\left(\theta^{*}-K-k_{c}\right)\left(\frac{\partial \varphi_{1}\left(y, Z+z_{c}\right)}{\partial y}+h^{\prime}(y)\right) \varphi_{1}\left(y, Z+z_{c}\right)\right) \\
& -\zeta\left(\left(\theta^{*}-K-k_{c}\right) \frac{\partial \varphi_{1}\left(y, Z+z_{c}\right)}{\partial z} \varphi_{2}\left(y, Z+z_{c}\right)\right)-y \varphi_{1}\left(y, Z+z_{c}\right), \\
\dot{Z}= & c \zeta-c\left(\theta^{*}-K-k_{c}\right) \varphi_{1}\left(y, Z+z_{c}\right)-c h(y)+\theta^{*} \varphi_{2}\left(y, Z+z_{c}\right), \\
\dot{y}= & -h(y)+\zeta+\left(K+k_{c}\right) \varphi_{1}\left(y, Z+z_{c}\right), \\
\dot{\zeta}= & -\mu(\zeta)+\left(\theta^{*}-K-k_{c}\right)\left(\frac{\partial \varphi_{1}\left(y, Z+z_{c}\right)}{\partial y}+h^{\prime}(y)\right)\left(K+k_{c}\right) \varphi_{1}\left(y, Z+z_{c}\right) \\
& +\left(\theta^{*}-K-k_{c}\right) \frac{\partial \varphi_{1}\left(y, Z+z_{c}\right)}{\partial z}\left(K+k_{c}\right) \varphi_{2}\left(y, Z+z_{c}\right),
\end{aligned}\right.
$$

which is parametrized by $\left(z_{c}, k_{c}\right)$. The linearization of the system (36) around the equilibrium $(K, Z, y, \zeta)=(0,0,0,0)$ is

$$
A_{c}=\left[\begin{array}{cccc}
0 & 0 & 0 & 0 \\
0 & 0 & g_{d} & c \\
0 & 0 & -h_{1}+k_{c} \psi_{c} & 1 \\
0 & 0 & g_{c} & -\mu_{1}
\end{array}\right],
$$

where $g_{d}=-c\left(h_{1}-k_{c} \psi_{c}\right)+\theta^{*}\left(\psi_{c c}-c \psi_{c}\right)$ and $\psi_{c c}=\psi^{2}\left(0, z_{c}\right)$. The matrix $A_{c}$ has three zero eigenvalues and one negative eigenvalue; therefore, the center manifold is three-dimensional. Now, we look for a coordinate transformation such that the coordinate axis become the linear eigenspaces of the linearization matrix $A_{c}$. The coordinate transformation is defined as

$$
\left(\begin{array}{c}
K \\
Z \\
y \\
\zeta
\end{array}\right)=\left[\begin{array}{cccc}
1 & 0 & 0 & 0 \\
0 & 1 & 0 & \frac{g_{d}-c \mu_{1}}{\lambda_{2}} \\
0 & 0 & -\frac{1}{\lambda_{2}} & 1 \\
0 & 0 & \frac{\lambda_{2}+\mu_{1}}{\lambda_{2}} & -\mu_{1}
\end{array}\right]\left(\begin{array}{c}
K \\
q \\
v \\
u
\end{array}\right) .
$$

This transformation keeps the $K$-coordinate. The equation for $K$ is easily obtained by substituting the new variables into (36). The transformation of 
the system (36) becomes

$$
\left\{\begin{array}{l}
\dot{K}=K_{20} v^{2}+K_{02} u^{2}+K_{11} v u+O(3), \\
\dot{q}=q_{10} v+q_{20} v^{2}+q_{02} u^{2}+q_{11} v u+K O(u, v)+q O(u, v)+O(3), \\
\dot{v}=v_{20} v^{2}+v_{02} u^{2}+v_{11} v u+K O(u, v)+q O(u, v)+O(3), \\
\dot{u}=\lambda_{2} u+u_{20} v^{2}+u_{02} u^{2}+u_{11} v u+K O(u, v)+q O(u, v)+O(3) .
\end{array}\right.
$$

Where

(40)

$$
\begin{aligned}
& K_{20}=\frac{\psi_{c}}{\lambda_{2}^{2}}\left[\left(\lambda_{2}+\mu_{1}\right)\left(\theta^{*}-k_{c}\right)\left(\psi_{c}+h_{1}\right)-1\right], \\
& K_{02}=\psi_{c}\left[\mu_{1}\left(\theta^{*}-k_{c}\right)\left(\psi_{c}+h_{1}\right)-1\right], \\
& K_{11}=\frac{\psi_{c}}{\lambda_{2}}\left[2-\left(\lambda_{2}+2 \mu_{1}\right)\left(\theta^{*}-k_{c}\right)\left(\psi_{c}+h_{1}\right)\right], \\
& q_{10}=\frac{c}{\lambda_{2}}\left[\left(\lambda_{2}+\mu_{1}\right)+\left(\theta^{*}-k_{c}\right) \psi_{c}+h_{1}\right]-\frac{\theta^{*}}{\lambda_{2}} \psi_{c c}, \\
& q_{20}=a_{1} k_{c}^{2}+a_{2} k_{c}+a_{3}, \quad q_{02}=b_{1} k_{c}^{2}+b_{2} k_{c}+b_{3}, \quad q_{11}=c_{1} k_{c}^{2}+c_{2} k_{c}+c_{3}, \\
& v_{20}=d_{1} k_{c}^{2}+d_{2} k_{c}+d_{3}, \quad v_{02}=e_{1} k_{c}^{2}+e_{2} k_{c}+e_{3}, \quad v_{11}=f_{1} k_{c}^{2}+f_{2} k_{c}+f_{3}, \\
& u_{20}=g_{1} k_{c}^{2}+g_{2} k_{c}+g_{3}, \quad u_{02}=l_{1} k_{c}^{2}+l_{2} k_{c}+l_{3}, \quad u_{11}=r_{1} k_{c}^{2}+r_{2} k_{c}+r_{3} .
\end{aligned}
$$

Here parameters $a_{i}, b_{i}, c_{i}, d_{i}, e_{i}, f_{i}, g_{i}, l_{i}, r_{i}$ for $(i=1,2,3)$ depend on $c, \theta^{*}$, and other parameters involved in Taylor expansion of functions $\psi^{1}, \psi^{2}, h$ and $\mu$. To perform this computation, it is assumed that we can expand functions $\psi^{1}, \psi^{2}$, $h$, and $\mu$ in their Taylor expansion with respect to $y, Z$ up to the order $N$ for $N$ large enough, that is,

$$
\begin{aligned}
\psi^{i}\left(y, Z+z_{c}\right) & =\sum_{m, n=0}^{N} \psi_{m, n}^{i} y^{m} Z^{n}+R_{N+1} \\
& =\psi_{00}^{i}+\psi_{10}^{i} y+\psi_{01}^{i} Z+\psi_{11}^{i} y Z+\cdots+R_{N+1}, \quad i=1,2, \\
h(y) & =h_{1} y+h_{2} y^{2}+\cdots+h_{N} y^{N}+R_{N+1} \\
\mu(\zeta) & =\mu_{1} \zeta+\mu_{2} \zeta^{2}+\cdots+\mu_{N} \zeta^{N}+R_{N+1}
\end{aligned}
$$

Then, coefficients stated in (40) can be computed using expansions (41)-(43).

Theorem 6.1. The closed-loop system (14) under the assumption of Theorem 3.2 does not generically possess any single wedge bifurcation.

Proof. We need to construct the center manifold for transformed system (39). Constructing the center manifold is lengthy, but straightforward; see, for example, [5]. The three-dimensional center manifold is tangent to the threedimensional hyperplane spanned by linear eigenspaces of three zero eigenvalues. In the new system of coordinates $(K, q, v, u)$ the center manifold is tangent to the $(K, q, v)$-hyperplane and is presented by $u=H(K, q, v)$ where 
$H(0,0,0)=0$. The center manifold is invariant. We have

$$
\dot{u}=\frac{\partial H}{\partial K} \dot{K}+\frac{\partial H}{\partial q} \dot{q}+\frac{\partial H}{\partial v} \dot{v} .
$$

Center manifold computation shows that the function $H$ cannot contain terms solely depends on $K^{n}$ for all $n \in \mathbb{N}$. The truncation of the center manifold up to the second order is

$$
u=\alpha v^{2}+\beta K v+\gamma q v
$$

Here,

$$
\begin{aligned}
& \alpha=-\frac{1}{\lambda_{2}} u_{20}+\frac{q_{10} k_{c} \psi_{01}^{1}}{\lambda_{2}^{4}}\left(\left(\lambda_{2}+\mu_{1}\right)+\left(\theta^{*}-k_{c}\right)\left(2 \psi_{c}+h_{1}\right)\right), \\
& \beta=-\frac{\psi_{c}}{\lambda_{2}^{3}}\left(\left(\lambda_{2}+\mu_{1}\right)+\left(\psi_{c}+h_{1}\right)\left(2 k_{c}-\theta^{*}\right)\right), \\
& \gamma=\frac{k_{c} \psi_{01}^{1}}{\lambda_{2}^{3}}\left(\left(\lambda_{2}+\mu_{1}\right)+\left(\theta^{*}-k_{c}\right)\left(2 \psi_{c}+h_{1}\right)\right),
\end{aligned}
$$

where

$$
\psi_{01}^{1}=\left.\frac{\partial \psi^{1}\left(y, Z+z_{c}\right)}{\partial Z}\right|_{y=0, Z=0} .
$$

The center manifold will have one of the following forms

$$
\begin{aligned}
u & =H(K, q, v)=q \widehat{H}(K, q, v)+v \widetilde{H}(K, q, v), \\
u & =H(K, q, v)=v \bar{H}(K, q, v) .
\end{aligned}
$$

If we consider the center manifold of the form (46), then the truncation of the center manifold reduction up to the second order will exhibit the generic Bogdanov-Takens bifurcation. Therefore, the origin of the reduced system will not have a non empty interior basin of attraction; more details about BogdanovTakens bifurcation is discussed, for example, in [4] and [5]. It is easy to check that if functions $\psi^{1}$ and $\psi^{2}$ contains no constants, then the center manifold will take the form (47), then the reduction of the system (36) to the center manifold takes the following form. Note that, generically, $q_{10} \neq 0$.

$$
\left\{\begin{array}{l}
\dot{K}=\hat{a} v^{2}+v g(K, q, v) \\
\dot{q}=v+\hat{b} v^{2}+v M(K, q, v) \\
\dot{v}=a K v+\hat{c} v^{2}+v L(K, q, v)
\end{array}\right.
$$

Here, the functions $g, M$ and $L$ contains nonlinear terms of order higher than two. Because of the specific configuration of the vector field in equations (48), we can apply the following singular time parametrization.

$$
t \mapsto t=\frac{1}{v} \tau
$$


The transformation (49) preserves the orbits of system (48), but the direction of time is reversed in the lower half-plane $v<0$; see [3] for more details for such singular time reparametrization. The resulting system is given by

$$
\left\{\begin{array}{l}
K^{\prime}=\hat{a} v+g(K, q, v), \\
q^{\prime}=1+\hat{b} v+M(K, q, v), \\
v^{\prime}=a K+\hat{c} v+L(K, q, v) .
\end{array}\right.
$$

where' denote the derivative with respect to $\tau$. Here the functions $g, M$ and $L$ have no linear terms. The origin is not an equilibrium of the system (50), but the $q$-axis is an invariant manifold. The organization of the flow around the origin will tell us about the local behavior of the origin for the original reduced system (48). The origin of the reduced system (48) cannot attract a non empty interior set. Suppose that the origin of (48) attracts a non empty interior set of initial conditions, let say $E$. For any initial value $\eta_{0}=\left(K_{o}, q_{0}, v_{0}\right) \in E$, there is a solution $\varphi_{t}\left(\eta_{0}\right)$, where $\varphi_{t}\left(\eta_{0}\right) \rightarrow 0$ as $t \rightarrow+\infty$. In the system (50), this orbit will be still there. However, the origin is not an equilibrium of the system (50). Therefore the corresponding orbit starting at $\eta_{0}$ contains the origin as a point located on that orbit. On the other hand, the $q$-axis of system (50) is invariant so that any solution starting on that axis near the origin pass through the origin. As the set $E$ has a non empty interior, the initial value $\eta_{0}$ can be chosen such that $\eta_{0}$ does not belong to the $q$-axis. This implies that there will be more than one solution passing the origin of the system (50) which contradicts with the existence and uniqueness theorems for differential equations. Hence, there will be no such a set as $E$ and the appearing of single-wedge bifurcation is ruled out. This proves the theorem.

We have seen that the single-wedge bifurcation does not occur. In summary, there are bad limit systems; however, they do not attracts a large set of initial conditions with non empty interior.

\section{Discussion}

The system (8) in the form of generalized strict feedback form defined by equations (6) and (7) are recursively partially stabilized with respect to $y$. The stability properties of stationary solution has been proved through two sets of assumptions stated in Theorems 3.2 and 3.3.

The success of stabilization is highly depended on $b$ to have a non zero known value. Here, we have one step and in accordance with equations (6) and (7) we have $b=B_{11}^{1}$. In general case of equation (6), at each step, $B_{11}^{i}$ and $B_{21}^{i}$ are matrix. There, at step $i$, all terms of $\bar{x}_{i}$-equation depend on $\bar{x}_{i}$ except $B_{11}^{i} y_{i+1}$ and $B_{21}^{i} y_{i+1}$ which depend on variable $y_{i+1}$. In the stabilization process, one take $y_{i+1}$ as the input for $\bar{x}_{i}$-equation and temporarily replace that with $u_{i}$. Later the variable $y_{i+1}$ will be recovered by change of variable $v_{i+1}=$ $y_{i+1}-u_{i}$. In mean time, $u_{i}$ needs to be designed carefully such that the aim of stabilization at step $i$ to be achieved. In this process, the success of stabilization 
is no longer simplified into a simple condition of being non zero. Instead, that requires a series of rank conditions to guarantee the stabilization of each step. For example consider the first step, $i=1$, we have $\dot{y}_{1}=B_{11}^{1} u_{1}+B_{12}^{1} z_{2}+\varphi_{1}^{1}$ and $\dot{z}_{1}=B_{21}^{1} u_{1}+B_{22}^{1} z_{2}+\varphi_{2}^{1}$; where $y_{2}$ is replaced with $u_{1}$. Now, the problem is to find a suitable $u_{1}$ such that $y_{1} \rightarrow 0$ as $t \rightarrow \infty$ while $z_{1}$ stay bounded. Such requirement usually leads to a rank condition on matrices, $B_{11}^{1}, B_{12}^{1}, B_{21}^{1}, B_{22}^{1}$. In a similar manner, this will proceed to complete the control of the whole system. A further comprehensive work is needed to establish these details which is beyond the scope of this paper.

We have also proceeded with the bifurcation analysis of the closed-loop system (14) under the assumption of Theorem 3.2. The relevant bifurcation diagrams have been presented. Then we focused on the quality of the adaptation process and specifically focused on single wedge bifurcation. The occurrence of single-wedge bifurcation can be quite complicated. We have found that such bifurcation does not generically take place in systems of the form (8) under the assumption of Theorem 3.2. The closed-loop system (14) under the assumptions of Theorem 3.3 does not necessarily possess a similar manifold of equilibria.

Acknowledgment. The author thanks S. B. Townley and H. M. Osinga for their valuable helps and comments. The author also wishes to thank anonymous referees whose comments and suggestions improved the quality of this paper.

\section{References}

[1] A. S. Andreyev, Investigation of partial asymptotic stability and instability based on the limiting equations, J. Appl. Math. Mech. 51 (1987), no. 2, 196-201.

[2] K. J. Åstrom and B. Wittenmark, Adaptive Control, Adison-Wesley, 1995.

[3] F. Dumortier and R. Roussarie, Geometric singular perturbation theory beyond normal hyperbolicity, Multiple-time-scale dynamical systems (Minneapolis, MN), 29-63, IMA Vol. Math. Appl., 122, Springer, New York, 2001.

[4] B. Fiedler and S. Liebscher, Takens-Bogdanov bifurcation without parameters and oscillatory shock profiles, Global analysis of dynamical systems, 211-259, Inst. Phys., Bristol, 2001.

[5] J. Guckenheimer and P. Holmes, Nonlinear Oscillations, Dynamical Systems, and Bifurcations of Vector Fields, Springer-Verlag, New York, 2nd edition, 1990.

[6] P. A. Ioannou and J. Sun, Robust Adaptive Control, Prentice-Hall, New Jersey, 1996.

[7] M. Krstić, Invariant manifolds and asymptotic properties of adaptive nonlinear stabilizers, IEEE Trans. Automat. Control 41 (1996), no. 6, 817-829.

[8] M. Krstić, I. Kanellakopoulos, and P. V. Kokotović, Adaptive nonlinear control without overparametrization, Systems Control Lett. 19 (1992), no. 3, 177-185.

[9] _ Nonlinear and Adaptive Control Design, John Wiley \& Sons, 1995.

[10] J. A. Leach, S. Triantafillidis, D. H. Owens, and S. B. Townley, The dynamics of universal adaptive stabilization: computational and analytical studies, Control Theory Adv. Tech. 10 (1995), no. 4, 1689-1716.

[11] I. G. Malkin, Theory of Stability of Motion, Izdat. Nauka, Moscow, 2nd edition, 1996.

[12] A. S. Oziraner, On asymptotic stability and instability relative to a part of variables, J. Appl. Math. Mech. 37 (1973), 659-665. 
[13] C. Risito, Sulla stabilitá asintotica parziale, Ann. Mat. Pura Appl. (4) 84 (1970), 279292.

[14] G. R. Rokni Lamooki and S. B. Townley, Adaptive partial stabilization for nondeterministic strict feedback form, Proc. 11th IEEE Int. Conf. on Meth. and Mod. in Aut. and Robo. MMAR, Poland, (2005), 249-254.

[15] G. R. Rokni Lamooki, S. B. Townley, and H. M. Osinga, Bifurcations and limit dynamics in adaptive control systems, Internat. J. Bifur. Chaos Appl. Sci. Engrg. 15 (2005), no. 5, 1641-1664.

[16] V. V. Rumyantsev, On the stability of motion with respect to the part of the variables, Vestnik Moscow. Univ. Ser. Mat. Mech Fiz. Astron. Khim. 4 (1957), 9-16.

[17] V. V. Rumyantsev and A. S. Oziraner, The Stability and Stabilization of Motion with Respect to Some of the Variables, Nauka, 1987.

[18] S. B. Townley, An example of a globally stabilizing adaptive controller with a generically destabilizing parameter estimate, IEEE Trans. Automat. Control 44 (1999), no. 11, 2238-2241.

[19] V. I. Vorotnikov, Partial Stability and Control, Birkhäuser, 1998.

[20] , Partial stability and control: The state-of-the-art and development prospects, Automation and Remote Control 66 (2005), no. 4, 511-561.

[21] L. Yang, S. A. Neild, D. J. Wagg, and D. W. Virden, Model reference adaptive control of a nonsmooth dynamical system, Nonlinear Dynamics 46 (2006), no. 3, 323-335.

SChOOL OF MATHEMATiCS

Statistics And Computer Science

COllege of SCIENCE

UNIVERSiTy OF TEHRAN, IRAN

E-mail address: rokni@khayam.ut.ac.ir 